\title{
UN TRYPTIQUE DE LA NOTION DE "DOSE » CHEZ L'HOMME IRRADIE
}

\author{
Roland LE GÔ * \\ (Manuscrit rę̧u le is février 1968)
}

\begin{abstract}
RÉSUMÉ
Nous avons voulu tenter d'introduire dans la notion de "dose " une signification biologique, afin que cette notion renouvelée de la dose puisse servir de référence au Médecin pour comparer entre elles les évolutions hématologiques, dans des cas différents d'irradiation humaine accidentelle.

Il nous a semblé pour cela nécessaire de développer les trois volets d'un tryptique, en montrant que l'on peut connaittre ou calculer successivement :

$\mathrm{I}^{\circ}$ une dose au fantôme corporel que l'on peut représenter spatialement par le Mannequin Transparent,

$2^{\circ}$ une dose physique à la moelle, calculée sous le nom de Dose Médullaire Homogène Moyenne (D.M.H.M.),

$3^{\circ}$ une dose exprimant les dommages aux cellules-souches, calculée sous le nom de Equivalent Biologique de Dose Médullaire (E.B.D.M.)

La représentation spatiale tridimensionnelle, dans le Mannequin transparent apporte des commodités d'observation directe de la répartition des doses physiques, en parti culier au niveau des organes critiques, la moelle principalement. Elle pose en outre les bases géométriques des calculs ultérieurs.

La D.M.H.M. est représentative d'une énergie physique totale, transmise à la moelle osseuse, du fait de sa distribution topographique. Elle ne peut servir de référence biologique pour les réactions hématologiques. Mais on peut vraisemblablement lui relier certains tests biologiques exprimant les lésions de "première collision cellulaire ", telles les lésions chromosomiques.

L'Equivalent Biologique de Dose Médullaire (E.B.D.M.) est une valeur dont le calcul est basé sur le taux de survie des cellules-souches de la moelle. Cette valeur cherche à être représentative de l'évolution hématologique, lorsque la moelle est l'organe critique. Elle introduit la notion de CELLULE CRITIQUE, en l'espèce la cellulesouche myéloīde, comme base nécessaire de comparaison entre les doses.

C'est cet E.B.D.M. qu'il faut prendre comme référence pour estimer les chances de survie - ce qui peut amener à des conclusions pronostiques et thérapeutiques.
\end{abstract}

* Département de la Protection Sanitaire. Centre d'Etudes Nucléaires de Fontenay-aux-Roses. B.P. $n^{\circ} 6,92$ - Fontenay-aux-Roses. 


\section{INTRODUCTION}

La conduite thérapeutique devant un cas accidentel d'irradiation importante chez l'homme est un problème souvent angoissant. Les bases sur lesquelles cette conduite s'établit progressivement sont de deux ordres : d'une part, les estimations de la dosimétrie physique; d'autre part, l'allure évolutive des retentissements biologiques et cliniques, dont on note au jour le jour la progression, notamment les courbes évolutives de la cytologie sanguine.

En ce qui concerne la dosimétrie physique, les estimations sont fournies par étapes successives. D'abord grossières et globales, elles sont progressivement affinées, grâce à des reconstitutions de l'accident, qui permettent d'utiliser de nombreux appareils de mesure, ainsi que des fantômes humains à l'intérieur desquels on peut disposer un grand nombre de dosimètres de natures diverses, sensibles aux différents rayonnements et aux différentes énergies.

A l'issue de ces mesures, les constatations dosimétriques permettent de se faire une opinion sur la dose reçue en différents points de l'organisme et de se rendre compte du caractère plus ou moins homogène de cette irradiation.

\section{EXPRESSION ACTUELLE DE LA DOSE \\ NÉCESSITÉ D’UNE REPRÉSENTATION DOSIMÉTRIQUE SPATIALE TRIDIMENSIONNELLE DU CHAMP DE DISTRIBUTION DE LA DOSE}

\section{LA DOSE DANS LES IRRADIATIONS DITES HOMOGÈNES}

Dans les cas d'irradiation homogène ou relativement homogène, la concordance obtenue dans l'ensemble des lectures fournies par les dosimètres permet d'aboutir en général à une valeur unique de la dose. Cette valeur est parfois explicitée en termes de dose gamma, et de dose neutrons, avec des sous-catégories: neutrons thermiques, épithermiques, rapides... gamma durs, rayonnements mous, etc.

Cette dose reste toutefois, soulignons-le, une notion purement physique. Cette grandeur ne peut prendre de sens au point de vue biologique, que si tout l'ensemble corporel a reçu en chaque point la même dose absorbée locale, c'est-à-dire si, en défininitve, l'irradiation a été réellement homogène.

Bien entendu, l'homogénéité réelle n'est jamais parfaitement vérifiée au cours d'une exposition accidentelle. Seules les irradiations globales thérapeutiques réalisent d'assez près cette condition. Dans les cas d'accidents, on peut seulement dire que l'on se rapproche, plus ou moins selon les cas, des conditions d'une irradiation homogène à la dose indiquée. On est alors autorisé à établir entre cette dose et l'évolution biologique, une corrélation. Celle-ci facilite la comparaison entre les évolutions biologiques observées sur différents accidentés.

Soulignons toutefois qu'il importe de ne jamais perdre de vue le fait que ce mode de langage n'est qu'une simplification par rapport aux faits réels, qu'il comporte toujours une distorsion vis-à-vis de la répartition véritable des doses locales dans l'organisme - et que, si l'on s'éloigne par trop des conditions qui 
définissent l'irradiation homogène, les valeurs de la dose dont on parle ne peuvent absolument plus servir de base d'intercomparaisons, entre les évolutions cliniques de différents patients.

\section{LA DOSE DANS LES IRRADIATIONS INHOMOGÈNES}

Les irradiations inhomogènes sont beaucoup plus nombreuses que les irradiations homogènes; elles sont réalisées par toutes les irradiations locales ou régionales pour traitement de maladies malignes - ainsi que par un certain nombre d'irradiations accidentelles.

Dans ces cas là, et en particulier en cas d'accident, la notion de " dose » exprimée sous la forme précédente, c'est-à-dire par une donnée unique, perd toute signification sur le plan biologique.

En effet, en chaque point de l'organisme irradié de manière inhomogène, la dose locale a une valeur individuelle. Pour avoir une vue suffisamment complète de la distribution de l'irradiation, il faut établir une représentation de la répartition des doses, exprimant en chaque lieu la valeur de la dose locale : c'est-à-dire, une représentation du champ de distribution de la dose.

Pour cela, il faut évidemment disposer du plus grand nombre possible de données dosimétriques locales recueillies à l'intérieur du corps — ou tout au moins, à l'intérieur du fantôme représentatif. Ce rôle est dévolu aux dosimétristes. Lorsque de telles données sont disponibles, on peut arriver à les regrouper, sous l'aspect d'une représentation spatiale tridimensionnelle du champ de dose.

\section{Représentation dosimétrique spatiale tridimensionnelle du CHAMp de DOSE}

Nous avons eu l'idée de cette représentation, à propos des problèmes posés par la dosimétrie, chez un irradié accidentel dont l'irradiation apparaissait comme très inhomogène sur la base des premières estimations dosimétriques. Nous l'avons réalisée par la technique du Mannequin Transparent en procédant comme suit :

Dans une première étape, les valeurs fournies par la dosimétrie physique, à partir des dosimètres inclus dans les tranches d'un fantôme en matière équivalenttissu (fig. I), ont été reportées sur des calques de chaque tranche. Sur chacun de ces calques, les valeurs de dose ont servi à établir des courbes isodoses. Cette étape n'est évidemment possible que si l'on dispose d'un nombre suffisant de valeurs de doses sur chaque tranche de fantôme. La surface incluse entre deux courbes isodoses est colorée dans un code correspondant à la dose.

Une fois les courbes et zones isodoses établies, la seconde étape consiste à en donner une représentation spatiale. Nous l'avons réalisée par un montage en plexiglas. Chaque calque représentant une tranche de fantôme et porteur du code-couleur des zones isodoses, est reproduit sous forme d'un gabarit en plexiglas mince découpé en vraie grandeur et sur lequel on reproduit les zones isodoses selon le code-couleur - ainsi que le contour des pièces squelettiques ou de tout autre organe.

L'assemblage des plaques de plexiglas est ensuite réalisé, dans la même géométrie que le fantôme réel : les plaques sont montées sur un axe commun et 


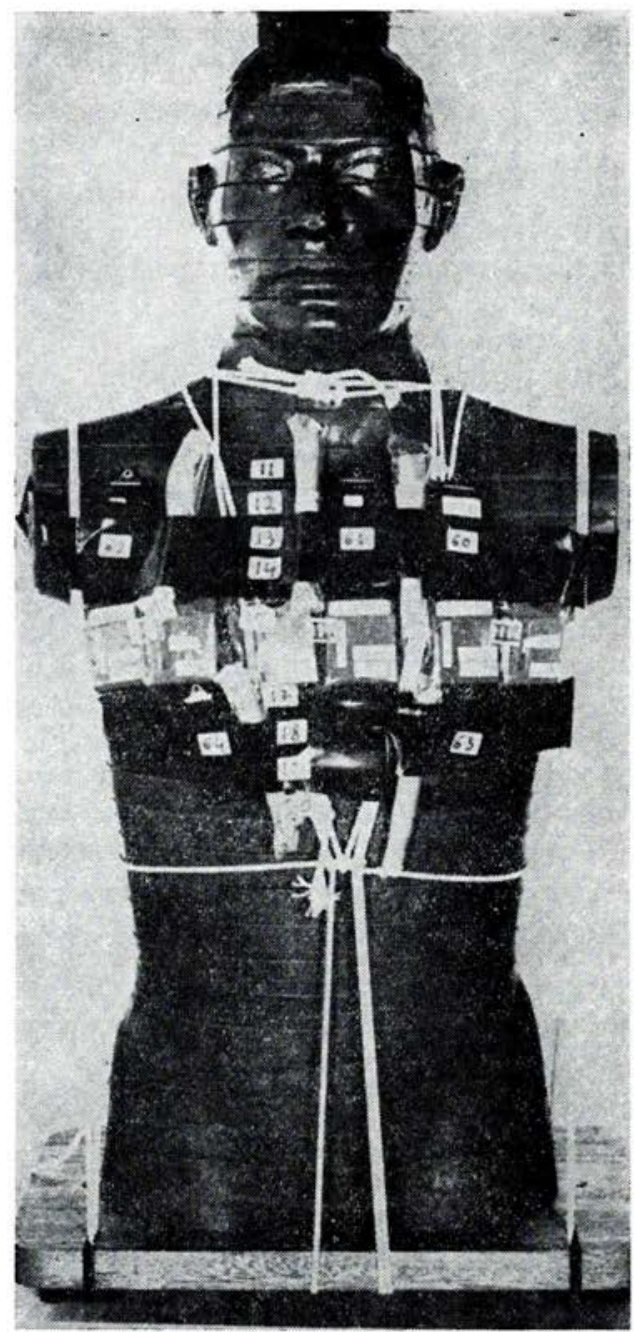

FIG. I. - Fantôme "Alderson » en matériau "tissu-équivalent » avec squelette incorporé. Ce fantôme permet l'introduction de dosimètre à l'intérieur de chacune des 34 tranches de $25 \mathrm{~mm}$ d'épaisseur dont il est constitué.

séparées les unes des autres par des entretoises, afin de reconstituer l'épaisseur des tranches du fantôme.

Cet assemblage définitif constitue le Mannequin Transparent (fig. 2), qui permet d'observer la répartition géométrique de l'irradiation dans l'ensemble de l'individu, dans une représentation spatiale tridimensionnelle. 


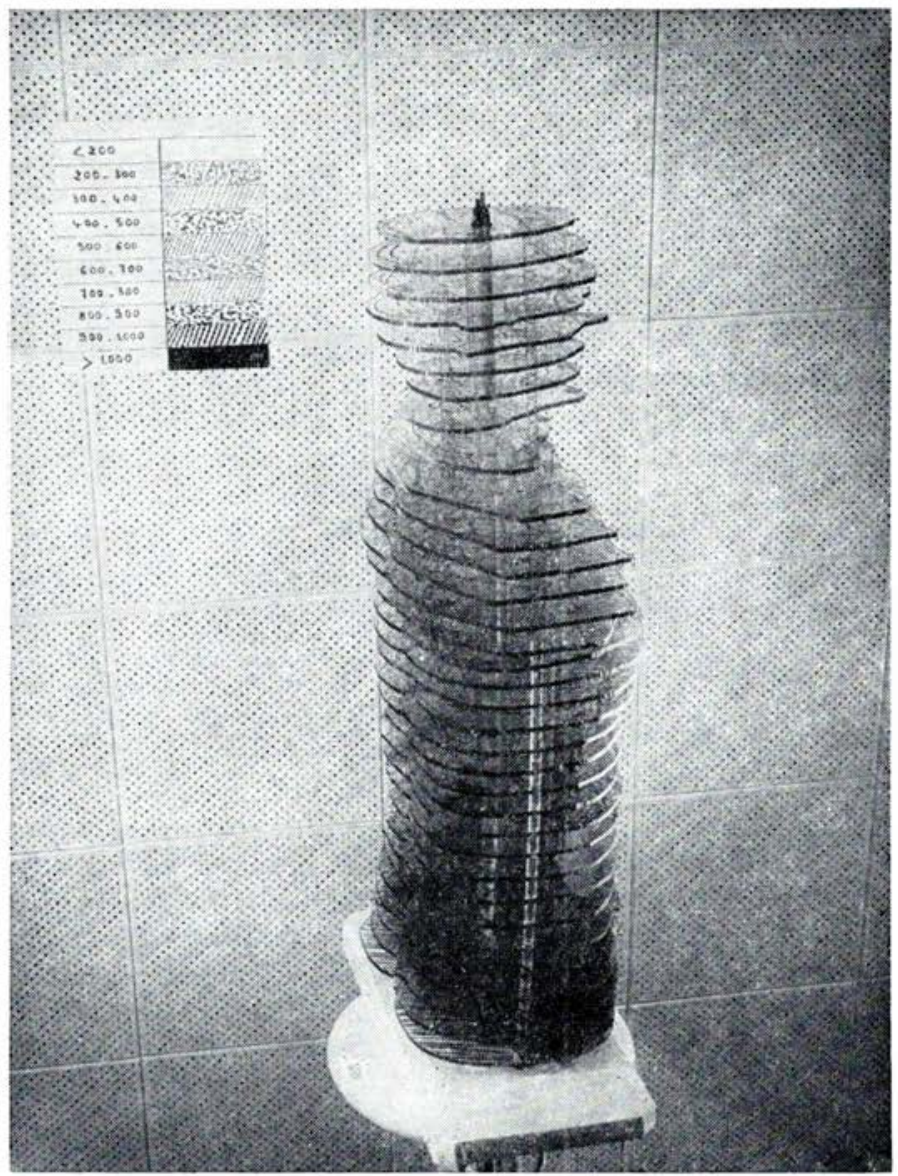

Fig. 2. - Mannequin Transparent.

Chacune des coupes en plexiglas de ro $\mathrm{mm}$ d'épaisseur est un gabarit en vraie grandeur de la tranche de numéro identique du fantôme précédent.

Sur chacune de ces coupes sont reproduits les contours du squelette et des organes critiques. Sur chacune d'elles également sont reproduites les zones isodoses: à chaque dose est affectée une couleur du code.

L'ensemble des tranches porteuses d'une même couleur constitue dans l'espace des " volumes isodoses 》 qui se matérialisent par transparence grâce à leur teinte.

\section{INTÉRÊT DU PROCÉDÉ}

L'intérêt de cette représentation est évident. Tout d'abord, elle apporte une vue spatiale de l'irradiation, telle qu'elle s'est réellement distribuée à l'intérieur de l'individu.

En ce qui concerne le flux d'irradiation lui-même, cette vue spatiale apporte des données quant à sa direction, à l'ouverture du faisceau, à l'atténuation en 
profondeur, ceci par le simple examen des tranches isodoses de même couleur, qui se constituent dans la masse du bonhomme transparent, par la superposition des gabarits porteurs de zones isodoses de même valeur.

En ce qui concerne l'irradiation des organes, on voit directement, en superposant leurs contours, tracés sur les coupes, dans quelles zones isodoses ils se trouvent inclus.

En particulier, l'intérêt principal de ce procédé, à notre sens, est de permettre une plus exacte appréciation de la manière dont a été irradiée la moelle osseuse, organe critique.

Au total, la représentation spatiale tridimensionnelle de la répartition des doses physiques, par le Mannequin Transparent, est la première étape vers une dosimétrie biologique. Elle permet de superposer la répartition spatiale des doses, à la répartition spatiale de l'organe-critique " moelle osseuse » dans le squelette.

Dès lors, sont posées les bases géométriques d'un calcul qui mènera à une dosimétrie médullaire, en prenant en considération, en chaque fraction de squelette, le pourcentage de moelle rouge présente et la dose reçue localement.

Ainsi pourra-t-on passer, du premier volet du tryptique de la notion de dose, à savoir la DOSE-FANTOME, au deuxième volet de ce tryptique : la DOSE A LA MOELLE.

\section{LA DOSE A LA MOELLE}

Le problème de la dosimétrie physique au moyen des fantômes ne peut résumer à lui seul la compréhension des dommages subis par un irradié. L'étape suivante dans cette compréhension est un problème de dosimétrie biologique, s'adressant expressément à l'organe-critique. C'est donc singulièrement un problème de dosimétrie médullaire, élément capital du bilan clinique et pronostique d'une irradiation. Notre propos est de poser les bases d'une estimation des dommages à la moelle, en établissant les notions de DOSES MÉdullaires.

Dans un premier temps, nous exposerons le principe de calcul d'une DOSE MÉDULLAIRE HOMOGÈNE MOYENNE.

\section{i. Calcul d'une dose médullaire homogène moyenne}

Notre objectif au cours de ce calcul est d'aboutir à une expression nouvelle de la notion initiale de dose, qui reste toutefois représentative du phénomène physique, du transfert total d'énergie au volume représenté par la moelle osseuse dans l'espace.

En effet, cette D.M.H.M. exprime l'énergie totale réellement reçue par ce volume de moelle au cours de l'irradiation inhomogène, mais en la supposant " théoriquement" répartie de façon " homogène ".

Par définition, la D.M.H.M. est donc la dose absorbée qui, si elle était distribuée de façon homogène à la moelle, correspondrait au même transfert d'énergie en Kerma que celui qui a été effectivement réalisé par l'irradiation inhomogène réelle : c'est donc la dose intégrale, divisée par la masse de la moelle.

Nous savons que cette estimation purement mathématique, d'une " énergie équivalente ", ne peut garder qu'un caractère de donnée physique. Nous savons qu'elle ne peut plus se maintenir sur le plan biologique. En effet, elle ne prend pas 
en compte le retentissement de l'inhomogénéité de répartition de la dose, sur le comportement local de chaque fraction élémentaire de moelle. Il y aura donc lieu de compléter cette première estimation en faisant intervenir des données qui tiennent compte de la réactivité cellulaire en fonction de la dose, pour chaque zone élémentaire de moelle osseuse.

\section{SignificAtion DE LA D.M.H.M.}

L'établissement de la D.M.H.M. n'est donc qu'une étape du tryptique de la notion de " dose ». Mais cette dose théorique possède aussi par elle-même une signification précise : elle permet d'imaginer l'évolution qui aurait pu être celle de l'irradié si la dose avait été distribuée de façon bomogène, pour une même énergie totale absorbée par la moelle.

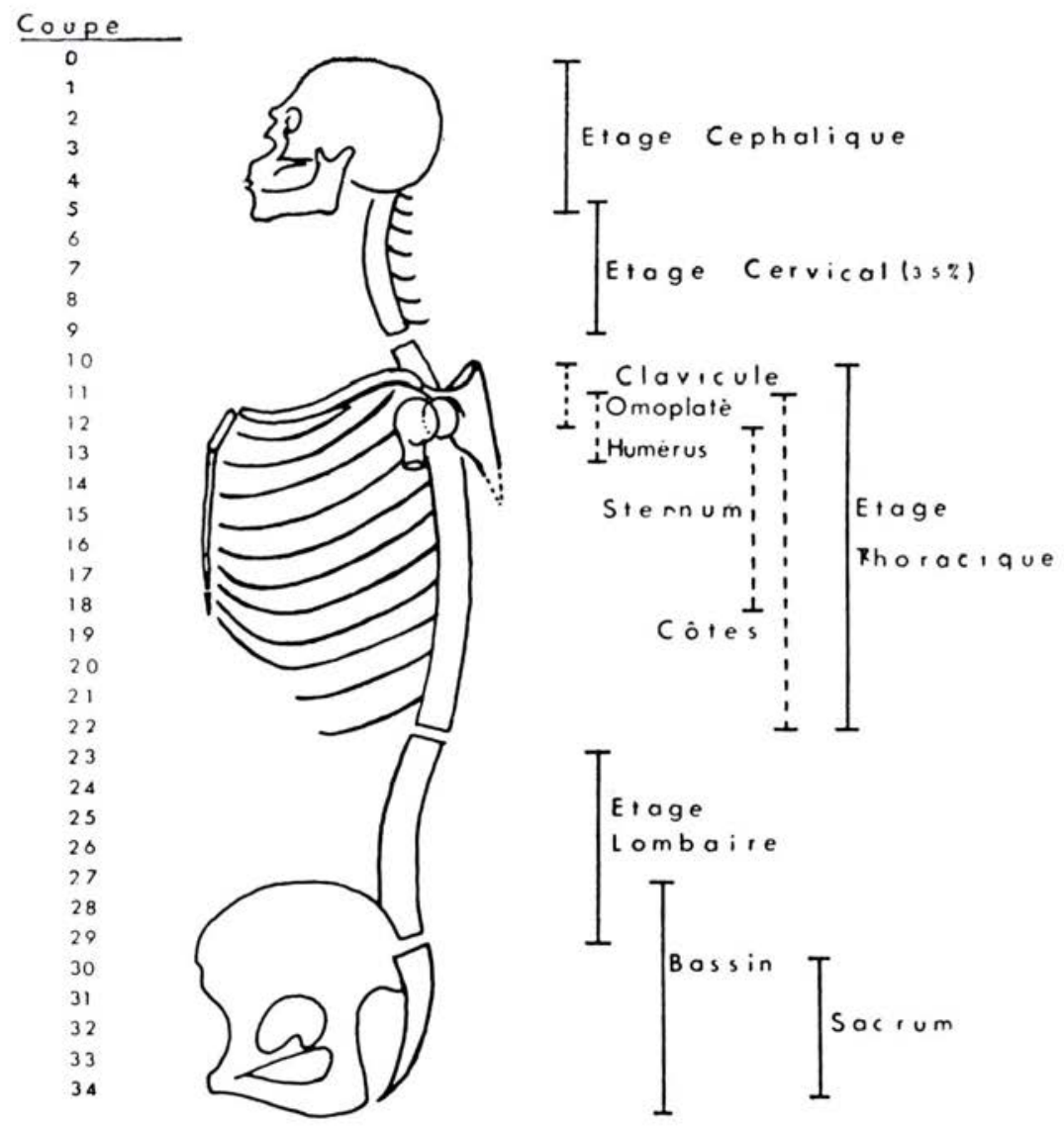

FIG. 3. - Schéma des régions anatomiques intéressées par les différentes coupes du fantôme. 


\section{Etapes du calcul de la D.M.H.M.}

Les étapes du calcul de la D.M.H.M. sont les suivantes:

Io Etablissement des zones isodoses sur le Mannequin Transparent;

$2^{0}$ Estimation sur chaque coupe du pourcentage de moelle incluse dans chaque zone isodose présente sur la coupe.

2.r. La moelle de chaque pièce squelettique (crâne, vertèbres, côtes, bassin, sternum, ceintures) représente : x p. Ioo du capital total de moelle osseuse.

2.2. Cette pièce osseuse est représentée dans le fantôme, sur les coupes de $n_{i}$ à $n_{j}$ (fig. 3).

2.3. Chaque coupe comporte donc (en moyenne) :

$$
\frac{x}{j-i+\mathrm{I}}=y_{i} \% \text { de moelle appartenant à cette pièce. }
$$

\section{TABLEAU 1}

Répartition de la moelle osseuse par régions

\begin{tabular}{|c|c|c|}
\hline \multicolumn{2}{|r|}{ ETAGE } & de la moelle \\
\hline Céphalique & partie antérieure $\ldots \ldots \ldots \ldots \ldots \ldots \ldots \ldots \ldots$ & 6,5 \\
\hline Cervical & $\begin{array}{l}\text { partie postérieure } \ldots \ldots \ldots \ldots \ldots \ldots \ldots \ldots \ldots \ldots \ldots \ldots \ldots \ldots \ldots \ldots \\
\text { vertèbres cervicales } \ldots \ldots \ldots \ldots \ldots \ldots\end{array}$ & $\begin{array}{l}6,5 \\
3,5\end{array}$ \\
\hline \multirow{5}{*}{ Thoracique } & vertèbres dorsales . & 14,9 \\
\hline & ceinture $\left\{\begin{array}{l}\text { Omoplate } \ldots \ldots \ldots \ldots \ldots \ldots \\
\text { Humerus } \ldots \ldots \ldots \ldots \ldots \ldots\end{array}\right.$ & 5,0 \\
\hline & ( Clavicule $. . . . \ldots \ldots \ldots \ldots . .$. & $\mathrm{I}, \mathrm{O}$ \\
\hline & côtes $\left\{\begin{array}{l}\text { moitié antérieure } \ldots \ldots \ldots \ldots \ldots \ldots \\
\text { moitié postérieure } \ldots \ldots \ldots \ldots \ldots \ldots\end{array}\right.$ & $\begin{array}{l}4,0 \\
4,0\end{array}$ \\
\hline & sternum $\ldots \ldots \ldots \ldots \ldots \ldots \ldots \ldots \ldots \ldots \ldots$ & 2,5 \\
\hline Lombo-sacré & 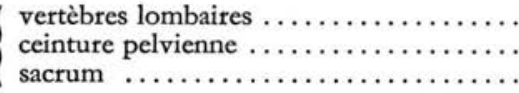 & $\begin{array}{l}11,0 \\
26,0 \\
14,0\end{array}$ \\
\hline \multicolumn{2}{|c|}{ Masse totale de la moelle pour l'homme adulte $\ldots \ldots \ldots \ldots$} & I $500 \mathrm{~g}$ \\
\hline
\end{tabular}

N.B. - On notera que ce tableau ne prend pas en compte la moelle incluse dans les parties distales des membres qui chez l'adulte ne représente qu'un pourcentage très faible. Par contre, dans les ceintures, scapulaire et pelvienne, est prise en compte la moelle épiphysaire supérieure de l'humerus et du fémur respectivement. 
2.4. Sur la coupe considérée, cette moelle est incluse et répartie entre $p$ zones isodoses. Chaque zone isodose recouvre donc la pièce osseuse considérée, pour un volume qui contient :

$$
\frac{y_{i}}{p}=Y_{i} \% \text { de moelle. }
$$

$3^{\circ}$ Ce $Y_{i} \%$ se trouve inclus dans une zone isodose de $d_{i}$ rads.

$4^{\circ}$ Calcul de la dose totale pondérée :

$$
D=\Sigma Y_{i} \cdot d_{i} \text {. }
$$

Cette dose totale pondérée représente la Dose Médullaire Homogène Théorique que nous recherchons.

\section{Estimation des pourcentages de moelle locale}

Nous nous servons d'estimations basées sur les travaux de Ellis [r], Lowrance et Latimer [2], Lisco [3], Maximow et Bloom [4] et groupées par Mechalr et Coll. [s]. Ces travaux donnent la répartition topographique de la moelle osseuse, par segments anatomiques osseux (Tableau I). Il faut ensuite transposer ces données au cas concret des coupes du Mannequin Transparent en établissant à quelles coupes (de $n_{i}$ à $n_{j}$ ) correspond chaque région définie dans le Tableau $\mathrm{I}$, et quelles pièces squelettiques se trouvent représentées dans chaque coupe.

Nous pouvons ensuite estimer le pourcentage de moelle présent dans chaque zone isodose d'une même coupe, puis établir le tableau de correspondance entre ces pourcentages $Y_{i}$ et la dose correspondante $d_{i}$, pour les $p$ zones isodoses présentes sur la coupe.

En principe, le tableau des calculs se présente ainsi :

\section{TABLEAU 2}

Estimation des doses élémentaires à la moelle

\begin{tabular}{|c|c|c|c|c|c|c|c|}
\hline$n_{i}$ & $d_{i}$ & $x$ & $j-i+\mathrm{I}$ & $y_{i}$ & $p$ & $Y_{i}$ & $d_{i} . Y_{i} \%$ \\
\hline 0 & $\begin{array}{l}400 \\
300\end{array}$ & 13 & 6 & $13 / 6$ & 2 & $13 / 12$ & $\begin{array}{l}400.13 / 12 \\
300.13 / 12\end{array}$ \\
\hline I & $\begin{array}{l}400 \\
300 \\
200\end{array}$ & & & & 3 & $13 / 18$ & $\begin{array}{l}400 \cdot 13 / 18 \\
300 \cdot 13 / 18 \\
200 \cdot 13 / 18\end{array}$ \\
\hline
\end{tabular}
pour l'étage anatomique...

où l'on a adopté les représentations suivantes :

$n_{i}=$ numéro de la coupe,

$d_{i}=$ isodose en rads,

$x=$ pourcentage de moelle totale de l'étage, 
$j-i+\mathbf{I}=$ nombre de coupes intéressées par l'étage anatomique, $y i=x(J-e+\mathrm{I})=$ pourcentage de moelle par coupe

$y_{i}=y_{i} \mid p=$ pourcentage de moelle par zone isodose,

$d_{i} . Y_{i}=$ dose pondérée par zone isodose, en p. Ioo.

Le calcul est effectué pour chacun des étages anatomiques, par la sommation des doses pondérées élémentaires $d_{i}$. $Y_{i}$.

Enfin, la somme des doses partielles pour chaque étage amène à une valeur globale de dose pondérée, qui est précisément la Dose Médullaire Homogène Moyenne que nous recherchons.

\section{Signification Biologique de LA “ DOSE MÉdullaire homogène MOYeNNE »}

Le mode d'établissement de la Dose Médullaire Homogène Moyenne n'a mis en jeu que deux sortes de données: des données physiques dosimétriques et des données anatomiques. La notion que l'on en retire ne peut donc être qu'une valeur exprimant le fait purement statique du transfert d'énergie à une structure anatomique: le contenu en moelle, du squelette.

On n'a fait entrer en jeu, dans le calcul, aucune donnée physiologique exprimant les dommages cellulaires en fonction de la dose. La valeur obtenue pour la D.M.H.M. reste donc essentiellement une estimation de nature physique.

On peut se demander cependant si sa signification ne peut pas aller au-delà d'une simple valeur physique. Certes, la transposition directe de cette valeur, en regard de l'évolution hématologique observée, n'est pas possible. Il n'est en rien équivalent en effet qu'un sujet ait reçu par exemple soo rads de façon homogène à l'ensemble de sa moelle, c'est-à-dire en D.M.H.M., ou bien qu'il ait reçu de façon inhomogène des doses réparties, suivant les régions corporelles, entre des valeurs très différentes, étagées par exemple entre $\mathrm{I}$ ooo rads et 200 rads.

Dans ce cas, quelle peut être la signification biologique de la D.M.H.M. ? Il semble que pour ce qui est de certains tests biologiques, qui ne mettent pas en jeu la réactivité cellulaire au cours du temps et les réparations qui s'ensuivent, la D.M.H.M. représente une estimation acceptable de la dose. Par exemple, il est probable que les lésions observées au niveau des chromosomes au cours de la première mitose, et dès les premières heures, (c'est-à-dire avant que n'interviennent les processus de cinétique des populations cellulaires qui vont modifier la persistance et la répartition de ces lésions de " première collision cellulaire "), ces lésions, donc, vont pouvoir être rattachées à la Dose Médullaire Homogène moyenne.

Il n'en va plus de même des lésions observées au niveau des cellules hématopoiétiques, dont la dynamique après irradiation ne peut être rapportée à la D.M.H.M. En effet, cette dynamique varie selon la dose locale, pour chaque fraction élémentaire de moelle. Le dépeuplement de la moelle au niveau des cellules-souches et, par suite, ses possibilités locales de régénération, ne peuvent être estimées globalement. Il est nécessaire de l'estimer par fractions élémentaires de moelle ayant reçu une dose déterminée et d'en regrouper les résultats pour l'ensemble des doses dans l'intervalle entre la plus élevée et la moins élevée de celles-ci. 
C'est ce que nous allons exposer dans le chapitre qui suivra, basé sur la survie des cellules-souches.

Résumons tout d'abord le point auquel nous sommes parvenus.

Dans l'établissement d'un tryptique de la notion " de dose » dont le premier volet est la Dose-fantôme, l'étape seconde est l'établissement d'une « Dose à la Moelle ", calculée de façon théorique, en tenant compte des données anatomiques de la répartition locale de la moelle et des données dosimétriques de la répartition locale de la dose.

Cette Dose Médullaire Homogène Moyenne a surtout une signification physique, car elle traduit l'énergie transmise à la totalité de la moelle.

Mais elle peut aussi servir de référence aux tests biologiques basés sur des lésions de "premier impact cellulaire ", telles que les lésions chromosomiques précoces.

\section{LA «DOSE AUX CELLULES-SOUCHES »}

Le calcul, exposé au cours du précédent chapitre, d'une Dose Médullaire Homogène Moyenne (D.M.H.M.), aboutit à une estimation statique de la situation après une irradiation inhomogène.

La valeur obtenue pour cette D.M.H.M.ne peut être comparée à des estimations biologiques, que lorsque celles-ci reflètent elles-mêmes la situation instantanée, immédiatement après l'irradiation; c'est-à-dire lorsqu'elles n'ont pas encore été influencées par les processus dynamiques de l'évolution biologique qui débutent dans le décours de l'irradiation.

$\mathrm{Si}$ maintenant nous voulons aboutir à une estimation qui rende compte réellement de l'évolution hématologique, il nous faut introduire dans nos calculs une donnée dynamique, qui se rapporte à la cinétique de la moelle osseuse en regard de la dose.

En effet ce qui importe essentiellement pour la survie d'un irradié, c'est de savoir si les régions corporelles relativement les moins endommagées - en tout cas à des doses inférieures à la DL so, contiennent suffisamment de pièces squelettiques riches en moelle active pour que l'on puisse espérer une survie spontanée.

\section{IMPortance DU FACTEUR TEMPS, EN REgARD DU FACTEUR DOSE}

Pour que l'on puisse espérer cette survie spontanée, il faut tout d'abord que le pourcentage de cellules-souches restées intactes dans la moelle la moins irradiée, permette un rétablissement spontané du capital initial de cellules-souches, par mitoses successives.

Mais il faut également - et ceci est capital - que cette restauration puisse s'accomplir dans un délai raisonnable. Cette condition fondamentale fait apparaître l'intervention du FACTEUR TEMPS dans le pronostic des chances de survie.

En effet, tout au long du délai occupé à la restauration du capital médullaire, l'irradié se trouve dans la période critique où, privé de leucocytes, privé de plaquettes, il ne peut absolument pas, par ses propres moyens, faire face à la survenue du moindre accident hémorragique ou infectieux. Il traverse, on le sait, une période de dénuement total sur le plan des défenses physiologiques. Il n'est donc pas exagéré de dire qu'à ce stade TOUT SE JOUE SUR LE FACTEUR TEMPS. 
Si le temps de restauration ne dure que quelques jours, cette période de dénuement peut être franchie assez simplement grâce à une attitude prophylactique rigoureuse, vis-à-vis des causes d'infection ou d'hémorragie.

$\mathrm{Si}$, au contraire, la restauration s'éternise, du fait du trop petit nombre des cellules-souches intactes et des mauvaises conditions de multiplication qu'elles rencontrent sur ce terrain fragilisé, alors la période de dénuement s'allonge de manière dangereuse; la menace de complications s'alourdit très vite et les méthodes prophylactiques deviennent impuissantes à enrayer, à elles seules, ces complications.

Il est donc essentiel d'aboutir à une notion de DOMmage Aux CEllulessouches et de la traduire en termes d'une DOSE HOMOGÈNE que nous appellerons ÉQUIVALENT BIOLOGIQUE DE DOSE MÉDULLAIRE (E.B.D.M.).

Notre propos est d'en poser les bases, dans ce troisième volet du tryptique de la notion de " dose ».

Etant donné que nous désirons que notre estimation puisse refléter l'évolution hématologique, il nous faut introduire dans nos calculs une donnée dynamique qui prenne en compte la cinétique de la moelle osseuse en regard de la dose.

Nous pensons que la donnée dynamique à choisir est le phénomène de dépeuplement médullaire en fonction de la dose : l'importance de ce dépeuplement étant à la base même de la cinétique de réparation hématologique.

Ceci étant admis comme postulat, on doit procéder, là encore, à l'estimation élémentaire du dépeuplement, pour chaque quantité élémentaire de moelle, se trouvant incluse dans une zone isodose déterminée.

En définitive, le calcul se ramène à l'estimation du pourcentage de cellulessouches restées viables dans chaque quantité élémentaire de moelle. Et la sommation de ces pourcentages élémentaires permet d'obtenir une estimation du nombre de cellules-souches restées viables dans la totalité de la moelle.

On peut alors, connaissant ce pourcentage, se référer aux courbes dose-effet de la survie des blastes et obtenir cette fois, en regard du taux de blastes survivants que l'on a calculé, la dose correspondante, qui est bien l'Equivalent Biologique de Dose Médullaire que l'on recherche.

\section{DÉfinition DE L'ÉQUiVALENT BIOLOGIQUe DE DOSE MÉdULlaire.}

L'E.B.D.M. est la dose absorbée qui, si elle est reçue en irradiation homogène aboutit à un dépeuplement équivalent des cellules-souches de la moelle osseuse. Ou si l'on veut, l'E.B.D.M. est la dose qui laisse le même pourcentage de blastes viables. En conséquence, elle a toutes les chances d'être assez bien représentative de l'évolution hématologique.

\section{Mode D'ÉtABlisSEMEnT DE L'E.B.D.M.}

Le problème qui se pose alors est de savoir à quelle courbe de référence on peut s'adresser pour connaître le taux de survie des cellules-souches de la moelle, en fonction de la dose. De telles courbes n'existent pas pour la moelle osseuse de l'homme. Cependant de nombreux travaux expérimentaux ont permis de connaître ce taux de survie chez l'animal, en particulier ceux de Till et McCulloch [6] [7]; ceux de LAjtha [8] [9]. L'animal d'expérience est la souris et les courbes expé- 
rimentales ont une forme linéaire en exprimant le logarithme du taux de survie, en fonction de la dose (fig. 4).

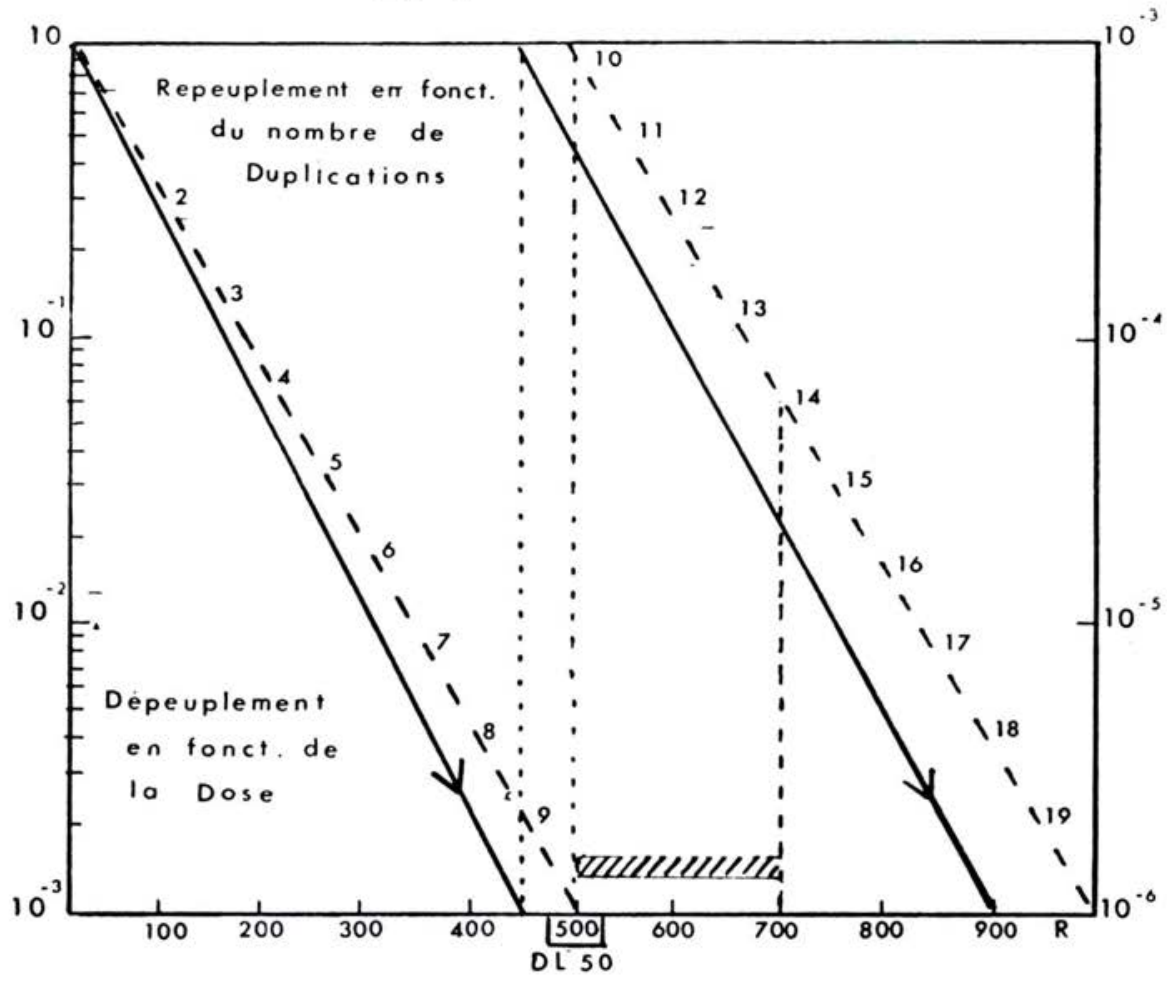

FIG. 4. - Courbe de dépeuplement des blastes en fonction de la dose, (établie d'après les résultats numériques de LAjTHA).

Evidemment, l'un des chefs principaux de notre autocritique a été la discussion du caractère licite de cette extrapolation qui nous fait assimiler les courbes de survie médullaire " des souris et des hommes").

Mais, outre le fait que nous ne disposons pas de courbes se rapportant à l'homme, il y a de bonnes raisons de penser que l'évolution de cellules aussi proches des caractères embryonnaires que le sont les blastes, doit peu différer quant aux réactions après irradiation, entre les espèces. Si cependant on admet que cette différence existe, ce n'est pas dans la forme évolutive de la courbe qu'elle doit apparaitre : cette courbe de dépeuplement est certainement aussi une droite logarithmique pour les blastes humains. La différence ne peut vraisemblablement apparaitre que dans une modification de la pente de cette droite.

Nous sommes conscients de cette différence mais nous pensons que le calcul, tel que nous l'avons exposé, mérite en tout cas de servir comme première approximation. Pour toutes ces raisons et faute de bases meilleures, nous nous sommes donc basés sur la courbe de dépeuplement des blastes, établie expérimentalement. 
Cette courbe donne le pourcentage des blastes survivants, pour chaque dose $d_{i}$, soit $B_{d i}$.

\begin{tabular}{c|c}
\hline \hline $\begin{array}{c}\text { Dose } \\
\text { en rads } \\
d_{i}\end{array}$ & $\begin{array}{c}\text { Pourcentage } \\
\text { de blastes survivants } \\
B_{d i}\end{array}$ \\
\hline 100 & $3,6 \cdot 10^{-2}$ \\
200 & $5,5 \cdot 10^{-2}$ \\
300 & $1,0 \cdot 10^{-2}$ \\
400 & $2,2 \cdot 10^{-3}$ \\
500 & $4,5 \cdot 10^{-4}$ \\
600 & $1,0 \cdot 10^{-4}$ \\
700 & $2,2 \cdot 10^{-5}$ \\
800 & $5,9 \cdot 10^{-6}$ \\
900 & $1,0 \cdot 10^{-6}$ \\
\hline \hline
\end{tabular}

Nous référant aux calculs établis précédemment, qui donnaient le pourcentage de moelle osseuse inclus dans chaque zone isodose, soit $Y_{i}$ et par ailleurs à la valeur de $B_{d i}$ pour chaque dose $d_{i}$, nous établissons le pourcentage de blastes restés viables dans chaque tranche de moelle isodose, puis la sommation de tous ces pourcentages (fig. 5 ).

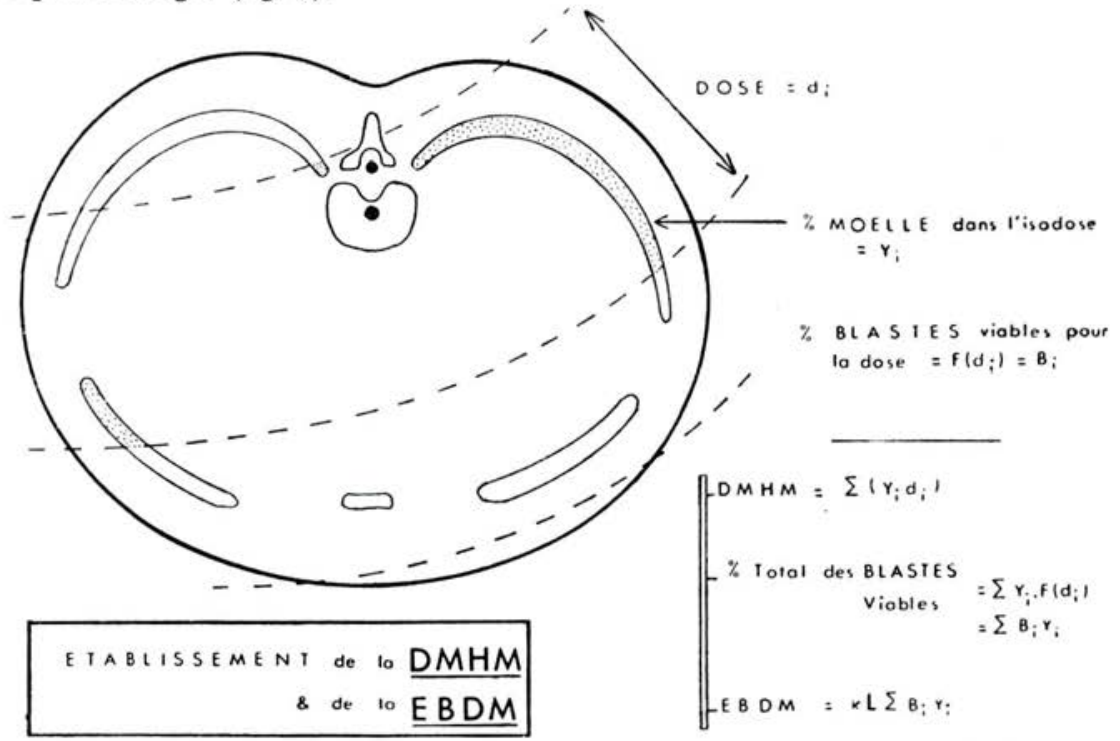

FIG. s. - Schéma d'une des coupes du Mannequin Transparent comportant la figuration : des isodoses $d_{i}$, du pourcentage de moelle présente $Y_{i}$, du pourcentage de blastes viables présents

$$
F\left(d_{i}\right)=B_{i} \text {. }
$$

Cette opération amène à un résultat qui représente le pourcentage réel de blastes restés viables dans la totalité de la moelle. Ce taux peut être, selon les cas, aussi faible que quelques pour cent, voir même quelques pour mille. 
Soit V, ce taux estimé de blastes “ viables ». Si l'on reporte la valeur de V, sur la courbe de dépeuplement des blastes (fig. 4), on constate que l'on peut lui faire correspondre une dose à la moelle dont la valeur est différente de celle de la Dose Médullaire Homogène Moyenne. Cette valeur nouvelle représente le troisième aspect de la notion de " dose » que nous recherchions : le troisième volet du tryptique.

Nous l'appelons l'Equivalent Biologique de Dose Médullaire. En effet, on peut dire, compte tenu de toutes les réserves faites précédemment, que cet E.B.D.M., distribué à la moelle en irradiation homogène, donne le même pourcentage de blastes survivants que la D.M.H.M. distribué d'une façon qui peut être fort inhomogène, selon les régions corporelles.

\section{Signification biologique de L'E.B.D.M.}

Alors que la D.M.H.M., comme nous l'avons vu, est représentative de l'énergie totale transmise à la moelle, elle ne peut servir de référence pour comparer entre eux des effets hématologiques.

Ceux-ci dépendent en effet en première approximation des dommages subis au niveau de la population des cellules-souches de la moelle. Partant du principe que l'on ne peut comparer que ce qui est comparable, c'est donc bien le dommage subi par la moelle, et plus précisément par les blastes, qui doit servir de référence pour définir dans ce domaine la véritable notion de la dose. Pour le Radiopathologiste et dans le domaine du Syndrome hématologique où la moelle est bien l'organe critique, la " dose " qui importe est donc celle qui exprime le dépeuplement des blastes: c'est la " dose aux cellules-souches ».

Ceci fait donc apparaître une transformation de la notion d'organe-critique, en une notion plus proche de la réalité physiopathologique : la notion de « CELLULE CRITIQUE ". C'est cette notion que tend à exprimer l'E.B.D.M., dans une première approche à ce problème.

\section{Différence dans les Chances de survie, ENTRe irRadiation INHOMOgène ET IRRADIATION HOMOGÈNE}

On peut traduire la différence constatée entre la Dose physique réellement absorbée par la moelle (D.M.H.M.) et la dose qui traduit le dommage réel au niveau des cellules-souches (E.B.D.M.), en termes de " chances de survie ».

La courbe de survie des populations humaines en fonction de la dose a été établie par Leaders, Williams et Schmidt [io] (fig.6). Si l'on reporte sur cette courbe les valeurs respectives de la D.M.H.M. et de l'E.B.D.M., on obtient les " chances de survie » correspondantes. Dans le cas d'irradiation inhomogène, les deux valeurs des chances de survie ne sont pas les mêmes. Elles peuvent être, par exemple, de 10 p. I0०, pour la première de ces valeurs, de $4 \circ \mathrm{p}$. 100 pour la seconde.

On peut considérer que la différence entre ces deux valeurs de chances de survie, exprime la différence évolutive entre une irradiation homogène et une irradiation inhomogène, pour la même énergie absorbée. On peut donc dire également que le fait qu'une irradiation soit inhomogène apporte un gain appréciable dans les chances de survie. Le " glissement » obtenu vers des chances de 
survie meilleures, peut être suffisamment marqué, pour que l'irradié puisse changer de catégorie pronostique, telles qu'elles ont été définies par de nombreux auteurs et particulièrement développées par Bond, Fliedner et ArCHAmbEAU (I I).

SURVIE DES POPULATIONS HUMAINES

EN FONCTION DE LA DOSE

$\%$ CHANCES de SURVIE

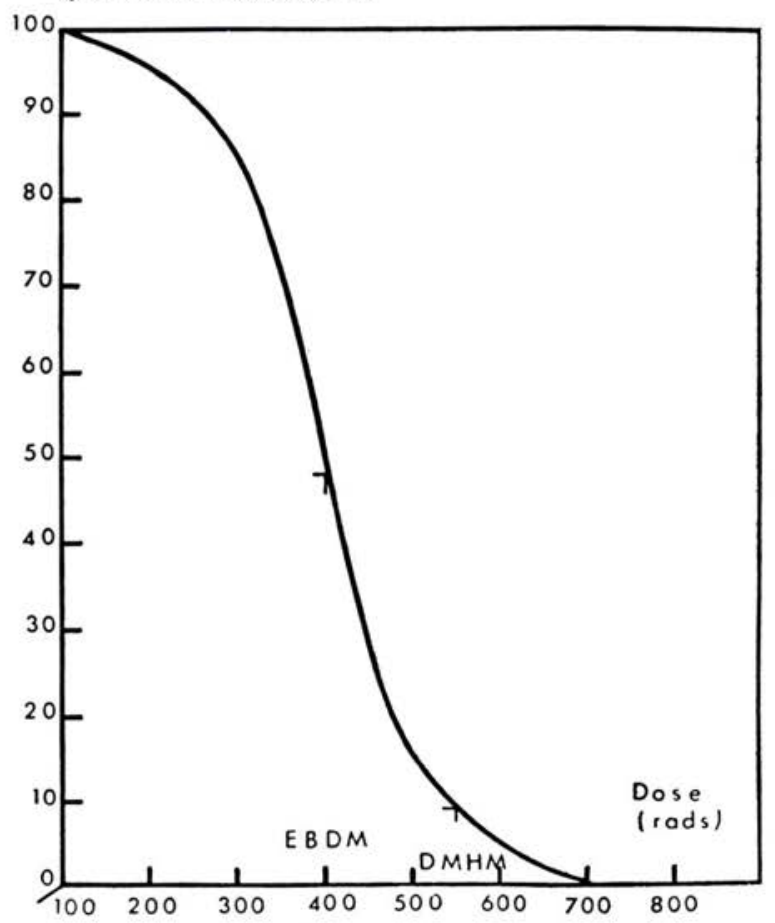

Frg. 6 - Courbe de survie des populations humaines, en fonction de la dose (établie d'après les résultats numériques de Leader Williams et Schmidt).

Cette constatation se montre spécialement importante lorsque le glissement fait passer l'accidenté de la catégorie survie improbable à la catégorie survie possible. En effet, elle peut avoir pour conséquence une prise de position diamétralement opposée, quant aux indications ou aux contre-indications d'une transplantation médullaire.

\section{CONCLUSIONS}

Nous avons voulu tenter d'introduire dans la notion de dose une signification biologique, en montrant que l'on peut connaître ou calculer successivement :

I $^{0}$ une dose au fantôme corporel, dont on peut représenter le champ de distribution tridimensionnel par un mannequin transparent, 
$2^{\circ}$ une dose physique à la moelle, calculée sous le nom de Dose Médullaire Homogène Moyenne,

$3^{\circ}$ une dose exprimant les dommages aux cellules-souches, calculée sous le nom de : Equivalent Biologique de Dose Médullaire.

L'E.B.D.M.cherche à être représentatif de l'évolution hématologique, lorsque la moelle est l'organe critique. Il introduit la notion de CELlule CRITIQUE, en l'espèce la cellule-souche myéloïde, comme base nécessaire de comparaison entre les doses.

C'est cet E.B.D.M. qu'il faut prendre comme référence pour estimer les chances de survie - ce qui peut amener à des conclusions pronostiques et thérapeutiques.

Certes, les bases biologiques du calcul de l'E.B.D.M. sont, pour l'instant, imparfaites. La connaissance du taux réel de survie des blastes de l'homme en fonction de la dose physique pourrait permettre de donner à cette notion un caractère plus rigoureux. Telle qu'elle est cependant, et avec ses imperfections, elle est une base de travail possible.

En outre, sa mise en application dans un cas humain concret d'irradiation nous a montré que l'E.B.D.M., tel que nous avons proposé de le calculer, permettrait d'aboutir à des conclusions non dénuées de sens.

\section{BIBLIOGRAPHIE}

[1] Ellis R.E. Phys. Méd. Biol., 5, 255-258 (1961).

[2] Lowrance E.W., Latrmer H.B.Aer. J. Anat, roi, 445, (1957).

[3] Lisco N. Argonne National Laboratory Rapport ANL-4253, 96, (1948).

[4] Maximow A.A., Bloom W. A textbook of bistology, Philadelphia, W.B. Saunders, (1948)

[s] Mechali D., Dousset M., Beau P., Le Grand J. Personnel Dosimetry for Radiation Accidents, Vienne, 8-12 mars 1965. Vienne, AIEA, 483-494.

[6] McCulloch E.A., Till J.E. The radiation sensitivity of normal mouse bone marrow cells, determined by quantitative marrow transplantation into irradiated mice. Radiat. Res., I 3, I I s-1 25 , (1960).

[7] Till J.E., McCulloch E.A. A direct measurement of the radiation sensitivity of normal mouse bone marrow cells. Radiat. Res., 14, 213-222, (1961).

[8] Lajtha L.G. Response of bone marrow stem-cells to ionizing radiation. Current topics in Radiation Research, Vol. I, 139-163, (1965).

[9] Lajtha L.G., Gilbert C.W., Porteous D.D., Alexanian R. Kinetics of a bone marrow stem-cell population. Ann. N-Y Acad. Sci., I13, 742-752, (1964).

[10] Leader-Williams, Schmidt J. The Practitioner 990, 594, (1960).

[iI] Bond V.P., Fliedner Th. M.,Archambeau J.O. Experience with radiation injury in man. In: Mammalian radiation letbality, a disturbance in cellular kinetics. Londres, Academic Press, $115-158$, (1965). 